\title{
Laparoscopic Total Colectomy with Transvaginal Extraction of the Colon and Ileorectal Anastomosis
}

\author{
Ziad Awad, MD, FACS \\ Department of Surgery, University of Florida, Jacksonville
}

\begin{abstract}
Background. The abdominal incision extraction site continues to be major source of morbidity after laparoscopic colectomy: mainly, incisional pain, wound infection, and incisional hernia. Also, in selected cases, it may delay initiating chemotherapy.

Methods. The video describes the technique of performing laparoscopic total colectomy, transvaginal removal of the entire colon, and intracorporeal ileorectal anastomosis in a 40-year-old woman with a sigmoid cancer and multiple endoscopically unresectable polyps throughout the colon. Computed tomography scan showed 2 liver lesions with carcinoembryonic antigen 138. Familial adenomatous polyposis gene analysis was negative. Six trocars (one $12 \mathrm{~mm}$ and five $5 \mathrm{~mm}$ ) were used. The whole colon was removed through the transvaginal route. The anvil was introduced through the vagina, and circular stapled ileorectal anastomosis was done.
\end{abstract}

Results. There were no intraoperative complications. The operating time was $210 \mathrm{~min}$. Blood loss was $20 \mathrm{~mL}$. The patient was discharged home on postoperative day 2 . Final pathology was T3N1bM1, and 2 of 23 lymph nodes were metastatic. All polyps were tubulovillous. She was commenced on chemotherapy 2 weeks after surgery. At 6-month follow-up, the patient was doing well, had no abdominal pain, and had no vaginal discharge or dyspareunia.

Conclusions. Natural orifice specimen extraction (NOSE) surgery can be added to the armamentarium of surgeons performing laparoscopic colon surgery. This technique may provide both an attractive way to reduce abdominal incision-related morbidity and a bridge to pure natural orifice transluminal endoscopic surgery (NOTES) colon surgery. Large-number patient data with long-term followup is needed.
Electronic supplementary material The online version of this article (doi:10.1245/s10434-014-3678-6) contains supplementary material, which is available to authorized users.

(C) Society of Surgical Oncology 2014

First Received: 12 August 2013;

Published Online: 18 April 2014

Z. Awad, MD, FACS

e-mail: ziad.awad@jax.ufl.edu 